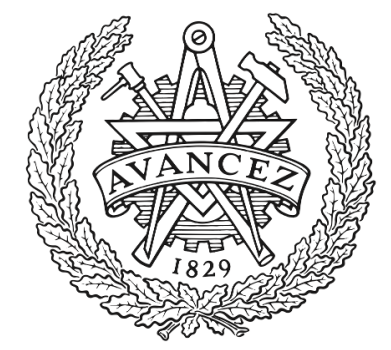

CHALMERS

UNIVERSITY OF TECHNOLOGY

\title{
Relativistically intense XUV radiation from laser-illuminated near-critical plasmas
}

Downloaded from: https://research.chalmers.se, 2023-04-26 11:38 UTC

Citation for the original published paper (version of record):

Blackburn, T., Gonoskov, A., Marklund, M. (2018). Relativistically intense XUV radiation from laser-illuminated near-critical plasmas. Physical Review A, 98(2).

http://dx.doi.org/10.1103/PhysRevA.98.023421

N.B. When citing this work, cite the original published paper. 


\title{
Relativistically intense XUV radiation from laser-illuminated near-critical plasmas
}

\author{
T. G. Blackburn,,$^{1, *}$ A. A. Gonoskov, ${ }^{1,2,3}$ and M. Marklund ${ }^{1}$ \\ ${ }^{1}$ Department of Physics, Chalmers University of Technology, SE-41296 Gothenburg, Sweden \\ ${ }^{2}$ Institute of Applied Physics, Russian Academy of Sciences, Nizhny Novgorod 603950, Russia \\ ${ }^{3}$ Lobachevsky State University of Nizhni Novgorod, Nizhny Novgorod 603950, Russia
}

(Received 25 January 2017; revised manuscript received 29 September 2017; published 23 August 2018)

\begin{abstract}
Pulses of extreme ultraviolet (XUV) light, with wavelengths between 10 and $100 \mathrm{~nm}$, can be used to image and excite ultrafast phenomena such as the motion of atomic electrons. Here we show that the illumination of plasma with near-critical electron density may be used as a source of relativistically intense XUV radiation, providing the means for novel XUV-pump-XUV-probe experiments in the nonlinear regime. We describe how the optimal regime may be reached by tailoring the laser-target interaction parameters and by the presence of preplasma. Our results indicate that currently available laser facilities are capable of producing XUV pulses with duration $\sim 10 \mathrm{fs}$, brilliance in excess of $10^{23}$ photons $/ \mathrm{s} / \mathrm{mm}^{2} / \mathrm{mrad}^{2}\left(0.1 \%\right.$ bandwidth), and intensity $I \lambda^{2} \gtrsim 10^{19} \mathrm{~W} \mathrm{~cm}^{-2} \mu \mathrm{m}^{2}$.
\end{abstract}

DOI: 10.1103/PhysRevA.98.023421

\section{INTRODUCTION}

There has been extensive demonstration of radiation sources in the extreme ultraviolet (XUV) to x-ray frequency range employing laser illumination of solid [1-4] and gaseous targets [5-7]. High-frequency radiation naturally arises in these interactions due to the nonlinear motion of electrons oscillating in the strong electromagnetic fields of an intense laser pulse. The generation efficiency and spectral properties of XUV sources are of paramount importance for applications in diagnostic imaging [8,9], the creation and study of warm dense matter [10,11], and for probing phenomena at the attosecond [12] and femtosecond scales [13].

Here we show that the XUV pulses emitted by laserilluminated near-critical plasmas can be relativistically intense, i.e., they are strong enough to accelerate electrons from rest to relativistic velocity in a single cycle. The physical origin of this emission is the near-micron scale oscillation of the plasma surface, which leads to the storage and reemission of a large fraction of the laser energy once per optical cycle. This ultimately compresses the reemission into an attosecond burst that has larger electric-field amplitude than the incident light $[14,15]$. The generation process is optimal when plasma with electron density between one and four times the relativistic critical density is irradiated at $60^{\circ}$ to the target normal. Under these conditions, $5 \%$ of the laser energy is converted to harmonics in the XUV frequency range and the reemitted pulse has peak intensity four times larger than the incident pulse. This mechanism is robust against the presence of preplasma and can, in fact, be enabled by it.

Laser-irradiated solid-density plasmas have attracted attention as sources of XUV light as, unlike gas-based sources, there is no upper limit on the input intensity [16]. For intensities $\sim 10^{16} \mathrm{~W} \mathrm{~cm}^{-2}$, below the relativistic threshold, coherent wake emission leads to harmonic generation in the ultraviolet [3]; at

\footnotetext{
*tom.blackburn@chalmers.se
}

higher intensities, experiments have demonstrated conversion efficiencies of $0.01\left(10^{-5}\right)$ to $\mathrm{x}$ rays with energy greater than $20 \mathrm{eV}$ (1 keV) [17], or $10^{-4}$ in the tens of $\mathrm{eV}$ [18] at $\sim 10^{19} \mathrm{~W} \mathrm{~cm}^{-2}$. The source of these high harmonics is the collective motion of electrons at the illuminated plasma surface, rather than atomic ionization and recombination.

Harmonic generation is commonly described by the relativistic oscillating mirror (ROM) model, in which the incident light is reflected at every instant of time within the wave cycle from a certain oscillating point $[19,20]$. The Doppler shift when this point moves back towards the observer at relativistic velocity leads to the increase in frequency of the reflected light. Models based on this assumption have given insight into polarization selection rules [20,21], the angular dependence of generation efficiency [22], and the power-law form of the intensity spectrum [23-27].

The descriptive power of the ROM model as formulated in [23] arises from the assumption of Leontovich boundary conditions at the oscillating surface, which imply instantaneous equality between incoming and outgoing energy flux and therefore bound the electric-field amplitude of the reflected light to that of the incident light. For highly overdense plasmas with steep density profile this assumption and its associated models work well.

However, with either increase of incident electric-field amplitude or decrease of plasma density, the assumption of instantaneous reflection begins to break down. Instead energy is first accumulated in a transient charge separation field when the laser radiation pressure displaces electrons from the plasma-vacuum boundary. Due to the relativistic nature of the motion, the displaced electrons are compressed into a thin layer, which reemits the stored energy when it propagates back towards the boundary. The parameter regime where this occurs provides an opportunity not only to compress the pulse in time but also to increase the reemitted intensity above that of the incident wave.

The relativistic electron spring (RES) model [15,28] describes these interactions by assuming that emission from the 
separated ions and electrons compensates the incident radiation within the plasma bulk, while allowing energy accumulation in the separation region. This assumption is weaker than that of instantaneous reflection, allowing for the description of a broader set of dynamics. The key result is that the generated radiation may have significantly higher electric-field amplitude than the incident light. In the ROM regime by contrast, the electric-field amplitude is strictly constrained to that of the incident light.

The RES model describes the plasma microdynamics in terms of the instantaneous displacement and velocity of a thin electron layer, the current of which generates coherent synchrotron emission (CSE). This has been studied in the context of creating reflected and transmitted attosecond pulses [29-32] and in thin-foil interactions [33,34]. In the RES regime the layer forms automatically, leading to the emission of attosecond [35] bursts with higher electric-field amplitude of the incident light [14,15,36], controllable ellipticity [37], as well as bright incoherent beams [38]. We will show in this work that the RES equations can be used to model the plasma dynamics and emission properties in the case that the plasma-vacuum interface is not perfectly sharp.

There has been considerable work devoted to optimizing XUV generation [39], including PIC simulation of few-cycle lasers [31,40], development of analytics $[15,22,28]$, and experiment. It has been shown, for example, that the denting of the plasma surface by the laser light pressure, which would otherwise increase the divergence of the high harmonics, may be compensated by tailoring the input laser pulse [41]. Simulations have also demonstrated the advantages of multiple reflection geometries [42] and specially designed waveforms for the incident light [43].

We use three dimensionless parameters to characterize the laser-plasma interaction: $a_{0}=e E /(m c \omega)$, the normalized amplitude of an electromagnetic wave with electric-field strength $E$ and angular frequency $\omega, \theta$, the angle between the laser wave vector and the target normal, and $S$, the ratio of the electron number density $n_{e}$ to that of the relativistic critical density $a_{0} n_{\text {cr }}$ [44]. Here $n_{\mathrm{cr}}=\epsilon_{0} m \omega^{2} / e^{2}$ is the nonrelativistic critical density and $e, m$ are the electron charge and mass and $c$ the speed of light, with $\epsilon_{0}$ the vacuum permittivity. The laser peak intensity $I_{0}=\frac{1}{2} a_{0}^{2} m c^{3} n_{\mathrm{cr}}$, or $I_{0}\left[10^{18} \mathrm{~W} \mathrm{~cm}^{-2}\right]=1.37 a_{0}^{2} /(\lambda[\mu \mathrm{m}])^{2}$ for wavelength $\lambda$. A convenient approximation for $S$ accurate within $5 \%$ is

$$
S=\frac{n_{e}}{a_{0} n_{\mathrm{cr}}} \simeq \frac{n_{23} \lambda_{\mu \mathrm{m}}}{\sqrt{I_{22}}}
$$

where $n_{23}$ is the electron number density in units of $10^{23} \mathrm{~cm}^{-3}$, $I_{22}$ is the laser intensity in units of $10^{22} \mathrm{~W} \mathrm{~cm}^{-2}$, and $\lambda_{\mu \mathrm{m}}$ its wavelength in microns.

Here $S$ is defined in terms of the bulk electron density of the plasma. However, all high-intensity laser systems exhibit finite contrast, leading to heating and expansion of the target by prepulse and pedestal light before the arrival of the main pulse. In this work we consider such effects by including a density ramp of scale length $L$ in front of the plasma bulk. We show that an effective $S$ parameter may be usefully defined in this scenario in Eq. (5). Throughout this work we consider semi-infinite plasmas as a model of thick targets; in the case that the target is a nanofoil with thickness $\ell$ and density equivalent

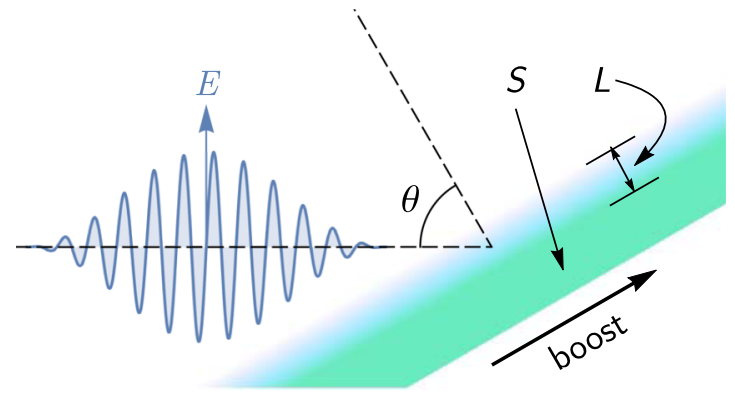

FIG. 1. Interaction geometry: a $p$-polarized laser pulse is incident onto a plasma slab with bulk electron density determined by the $S$ parameter and linear density ramp with scale length $L$.

to $S$, a parameter $s=S \omega \ell /(2 c)$ may be defined that plays an equivalent dynamical role [24].

The geometry under consideration is shown in Fig. 1. In all the interaction scenarios we consider, the laser is $p$ polarized. It is possible to simplify the problem to one with only a single spatial dimension by boosting by $c \sin \theta$ in the direction parallel to the plasma surface [45]. Neglecting the transverse intensity variation of a focused laser pulse, in this frame the laser may be treated as a plane wave with electric-field amplitude $a_{0} m c \omega \cos \theta / e$ and angular frequency $\omega \cos \theta$, which is normally incident on a plasma with bulk density $n_{e} / \cos \theta$ and streaming velocity $c \sin \theta$ perpendicular to the target normal.

The figures of merit we use to characterize XUV generation are the effective normalized amplitude $a_{\text {eff }}$, defined in Eq. (3), and the conversion efficiency $\eta_{\mathrm{XUV}}$, the fraction of the incident laser energy re emitted to light in the XUV frequency range (i.e., between wavelengths of 10 and $100 \mathrm{~nm}$ ) across the entire pulse train. To assess the viability of using the RES regime as a source of intense XUV radiation, we consider here plasmas with electron density between 0.25 and 20 times the relativistic critical density and laser pulses with intensity and duration that can achieved in currently available laser systems.

\section{XUV GENERATION MECHANISM}

The key property of the laser-plasma interaction at nearcritical densities is that the electric-field amplitude of the reemitted light can be much larger than that of the incident light. One may see how advantageous this is for XUV generation in the following way: to keep the total energy of the pulse the same, an increase in the electric-field amplitude by a factor $f=E_{r} / E_{0}$ must be accompanied by a reduction in the duration by a factor $f^{2}$. The emission of one such short pulse per optical cycle indicates that laser energy is efficiently converted to high harmonics. Furthermore, the fact that $a_{0} \propto$ $E / \omega$ raises the possibility of generating relativistically intense $\mathrm{XUV}$ light, as the increase in $\omega$ due to high harmonic generation may be partially compensated by the electric-field amplitude increase that occurs at near-critical densities.

Results of 1D PIC simulations using the spectral code ELMIS [46] that confirm this are shown in Fig. 2. For this set of simulations we consider laser pulses with laboratory-frame electric field $\left(a_{0} m c \omega / e\right) \sin \phi \cos ^{2}(\phi / 8)$ (for phase $|\phi|<4 \pi$ ) and wavelength $1 \mu \mathrm{m}$ incident at $\theta=60^{\circ}$ onto preplasma-free targets. The duration of such a pulse is $5 \mathrm{fs}$ [full width at 

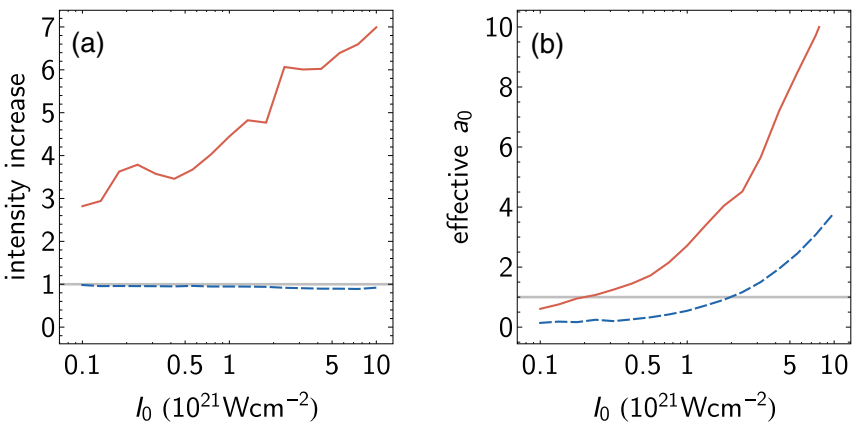

FIG. 2. (a) Intensity increase and (b) the effective $a_{0}$ of the reemitted pulse when plasma with $S=1$ (red) and $S=20$ (blue, dashed) is illuminated by a laser pulse with peak intensity $I_{0}$, duration $5 \mathrm{fs}$, and wavelength $1 \mu \mathrm{m}$ at $60^{\circ}$ to the target normal.

half maximum (FWHM) of the intensity profile]. The boosted frame coordinate system is defined such that the laser phase $\phi=\omega \cos \theta\left(t^{\prime}-z^{\prime} / c\right)$ and that its electric-field vector lies along $x^{\prime}$, corresponding to $p$ polarization. The plasma has initial density $n_{e, 0}=S a_{0} n_{\mathrm{cr}} / \cos \theta$, following Eq. (1), and streams along $x^{\prime}$ with velocity $v_{x^{\prime}, 0} / c=-\sin \theta$. It has temperature $100 \mathrm{eV}$ and is represented by 200 macroelectrons and ions $\left(\mathrm{C}^{6+}\right.$, i.e., $\left.Z / A=0.5\right)$ per cell of size $\Delta z^{\prime}=\lambda /(1800 \cos \theta)$.

Let us compare the near and supercritical cases $S=1$ and $S=20$. Figure 2(a) shows that the peak intensity of the reemitted pulse is more than twice that of the incident light for all intensities in the range $10^{20}$ to $10^{22} \mathrm{~W} \mathrm{~cm}^{-2}$ if the plasma density satisfies $S=1$. If instead $S=20$, the peak intensity is always bounded by that of the incident pulse. In both cases the reemitted radiation is broadband, though we will show later that the conversion efficiency to XUV (harmonics from 10th to 100th order) is larger for lower $S$. First we discuss how the intensity increase leads to enhanced $a_{0}$ for $S=1$.

A electromagnetic wave is relativistically intense if it can accelerate electrons from rest to relativistic velocity within a single cycle. Given the electric field of the reemitted pulse $E_{r}$ as a function of phase $\phi$, the transverse four-velocity of an electron is

$$
u_{\perp}=-\int \frac{e E_{r}(\phi) \lambda}{2 \pi m c^{2}} d \phi,
$$

according to the conservation of transverse canonical momentum. We define the effective $a_{0}$ as

$$
a_{\mathrm{eff}} \equiv \max u_{\perp} .
$$

Here $\lambda$, the wavelength of the incident light, appears for convenience because it defines the interval between emission of individual attosecond pulses. Neither $u_{\perp}$ nor $a_{\text {eff }}$ depend directly on $\lambda$, because the phase $\phi$ contains a factor of $1 / \lambda$. The reemitted pulse is relativistically intense in the XUV range if $a_{\text {eff }}>1$ when it is calculated using an $E_{r}(\phi)$ that has been filtered to exclude wavelengths outside the range $10 \mathrm{~nm}<\lambda<100 \mathrm{~nm}$. [For a monochromatic plane wave with electric-field amplitude $a_{0} m c \omega / e$, Eq. (3) reduces to $a_{0}$ as expected.]

We show the effective $a_{0}$ of the reemitted XUV radiation in Fig. 2(b) as function of incident intensity. It is larger for $S=1$ than for $S=20$ across the whole range of intensities. For $S=1$
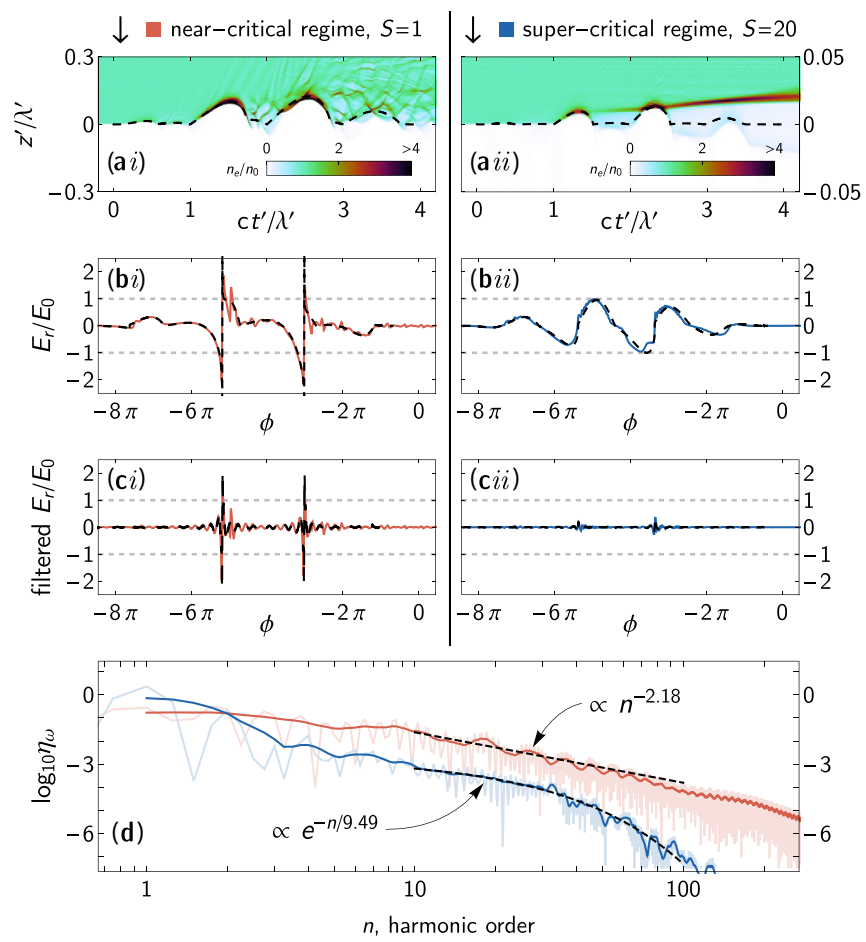

FIG. 3. Comparison of XUV generation in laser illumination of near- and supercritical plasmas: (a) the electron density in the boosted frame (color scale) and the RES model prediction for the electron displacement (dashed); (b) the laboratory-frame electricfield amplitude of the reemitted light $E_{r}$ relative to the peak initial amplitude $E_{0}$ (colors) and RES model predictions of the same (black, dashed); (c) as in (b), but filtered to the XUV frequency range; (d) the spectral conversion efficiency from simulations: full (lighter colors), smoothed over the width of a single harmonic (darker colors), and analytic fits for the XUV frequency range (black, dashed).

the relativistic regime is reached for $I_{0}>2 \times 10^{20} \mathrm{~W} \mathrm{~cm}^{-2}$, an order of magnitude less than that required if $S=20$. At $10^{22} \mathrm{~W} \mathrm{~cm}^{-2}$, the current intensity frontier [47], $a_{\text {eff }}$ reaches 10 , raising the prospect of scaling relativistic laser-plasma phenomena from optical to XUV wavelengths [48].

The origin of the intensity increase in the near-critical regime is the increased amplitude of the plasma surface oscillation. To demonstrate this, we compare the results of PIC simulations of near-critical $(S=1)$ and supercritical $(S=20)$ plasmas that are illuminated by a laser pulse with intensity $I_{0}=10^{21} \mathrm{~W} \mathrm{~cm}^{-2}$ at an angle of incidence $\theta=60^{\circ}$. We also compare these findings to the theoretical predictions of the RES model by solving the RES equations numerically. No fitting or free parameters are used; for details and more benchmarking against simulations, see [28].

Figure 3(a) compares the electron density in the boosted frame as a function of axial displacement $z^{\prime}$ and time $t^{\prime}$, normalized to the wavelength in the boosted frame $\lambda^{\prime}=\lambda / \cos \theta$. The oscillations arise because the laser radiation pressure pushes electrons into the plasma, gathering them into a thin sheet of high charge density at $z_{e}^{\prime}$. The uncompensated ion current in the region $0<z^{\prime}<z_{e}^{\prime}$ sets up electrostatic and magnetostatic fields, the former exerting a force on the electron density spike that balances and then exceeds the radiation pressure. The fact 
that this force is proportional to the displacement $z_{e}^{\prime}$, assuming the downstream region is entirely cleared of electrons, is the origin of the name "relativistic electron spring" [15]. We see that the RES equations predict both the scale and period of the instantaneous displacement of the electrons very well. At $S=20$, a discrepancy arises towards larger $t^{\prime}$ that is due to ion motion; the breakup of the plasma surface leads to the last half-wavelength of the pulse being reflected in a region of lower density at $z^{\prime} / \lambda^{\prime} \simeq-0.01$.

During the phase of the motion when the electrons return towards $z^{\prime}=0$, they acquire kinetic energy from the plasma fields, reaching high $\gamma$. When their transverse velocity changes sign, at which moment in the laboratory frame they propagate towards the observer along the specular direction, a largeamplitude burst of radiation is emitted. This is shown in Fig. 3(b): for $S=20$, the waveform acquires a nonsinusoidal shape but the electric-field amplitude never exceeds the incident $E_{0}$, whereas for $S=1$ for the waveform is characterized by sharp transitions between positive and negative field and amplitude increase $\max \left(E_{r} / E_{0}\right) \simeq 2$.

This difference is clearly demonstrated when the reemitted light is filtered to the XUV frequency range using a rectangular bandpass filter that is nonzero for wavelengths between between 10 and $100 \mathrm{~nm}$, as shown in Fig. 3(c). We find that for $S=1$ the reemitted attosecond pulses have electric-field amplitude twice that of the incoming light, which corresponds to a peak intensity of $4.0 \times 10^{21} \mathrm{~W} \mathrm{~cm}^{-2}$. For $S=20$, by contrast, the peak intensity of the filtered pulses is $1.1 \times 10^{20} \mathrm{~W} \mathrm{~cm}^{-2}$. We would expect $a_{\text {eff }}$ to be larger in the former case by a factor of $\sim 6$ (the ratio of the pulse electric-field amplitudes); as Fig. 2(b) shows $a_{\mathrm{eff}}$ to be five times larger for $S=1$, this is reasonably accurate.

As the RES equations predict the temporal evolution of the current carried by the electron layer, we can use them to extract the waveform of the reemitted radiation; the theoretical and simulation results are in good agreement for the total [Fig. 3(b)] and spectrally filtered [Fig. 3(c)] electric-field amplitudes. (The waveforms here and in Sec. III are compared when they have moved four wavelengths from the plasma surface.) The overestimation of the peak value of $E_{r}$ for $S=1$ is a consequence of a singularity in the RES equations that occurs at the instant the transverse velocity changes sign [28]. This does not occur in the PIC simulations because the electron layer is guaranteed to move with speed less than $c$.

The fact that the amplitude of the electric field, when filtered to XUV frequencies, is larger for $S=1$ than in $S=20$ means that, in the former, there must be more energy carried in that frequency range and therefore higher conversion efficiency. We can confirm this by comparing the spectral conversion efficiencies (energy carried by harmonic $n$ per unit energy of the incident pulse) we obtain from the simulations. Note that the analytical fits we provide to the intensity spectrum in the XUV frequency range deviate from the predictions of the standard ROM and CSE models ( $n^{-8 / 3}$ and $n^{-4 / 3}$, respectively); however, power-law decay exponents from $-7 / 3$ to $-5 / 3$ have been reported, as has their sensitivity to the choice of frequency range [26]. As the main purpose of our analysis is to find when the reemitted XUV pulses can be relativistically intense, the most important quantity is the magnitude of the intensity spectrum; Fig. 3(d) shows that the spectral conversion efficiency is at least an order of magnitude greater for $S=1$ than for $S=20$ across the range $10<n<100$. Specifically, we find that the total XUV conversion efficiency $\eta_{\mathrm{XUV}}$, obtained by integrating over this range, is $19.2 \%$ for $S=1$ and $0.67 \%$ for $S=20$. The explains why the effective $a_{0}$ shown in Fig. 2(b) exceeds unity at a lower laser intensity if the plasma density is near critical.

\section{PREPLASMA}

Thus far we have considered interactions with plasma targets where the bulk electron density is near critical. This is experimentally changing, but possible with the use of cryogenic, aerogel, or porous foam targets [49]. An alternative approach would be to exploit the finite contrast of a high-intensity laser pulse, because prepulse causes heating and expansion of the target and therefore the laser-plasma interaction takes place within a density ramp with scale length $L$. In this section we show that $L>0$ can enhance XUV generation for the same reason explored in Sec. II, by reducing the apparent density of the plasma.

To do so, we introduce an effective $S$ parameter that characterizes the laser-plasma interaction when $L$ is nonzero. We take the preplasma to be a linear density ramp with scale length $L$, i.e., $n_{e, 0}=\left(S a_{0} n_{\mathrm{cr}} / \cos \theta\right)\left(1+z^{\prime} / L\right)$ for $-L<z^{\prime}<0$. The laser pulse will penetrate the density ramp up to the point $z^{\prime}=z_{\max }^{\prime}$ where the electrostatic force of the charge separation field is balanced against the magnetic forces arising from the laser itself and the uncompensated ion current. Assuming that all electrons in the region $z^{\prime}<z_{\max }^{\prime}$ are swept forward, we find that

$$
\frac{z_{\max }^{\prime}}{L}=-1+\sqrt{\frac{\lambda(1+\sin \theta)}{\pi L S}},
$$

where $S$ is calculated from the bulk plasma density and $\lambda$ is the laser wavelength in the laboratory frame.

The value of $S$ that appears in Eq. (4) is calculated using the peak normalized amplitude of the laser pulse $a_{0}$. The instantaneous penetration depth may be estimated by replacing $S$ with its instantaneous value $S / \cos ^{2}[\phi /(2 N)]$, where $N$ is the (arbitrary) number of cycles corresponding to the pulse duration. Averaging the penetration depth over the interval $-N \pi<\phi<N \pi$ gives us an average density which may be used to define an effective $S$ parameter for the interaction. Assuming that the maximum penetration depth is smaller than the preplasma scale length $L$, we find

$$
S_{\text {eff }}=\sqrt{\frac{4 S \lambda(1+\sin \theta)}{\pi^{3} L}}, \quad L>\frac{(1+\sin \theta) \lambda}{\pi S} .
$$

For smaller $L$, the laser pulse breaks through the density ramp and the bulk plasma is the source of the reemitted radiation; in this case we would have $S_{\text {eff }}=S$. A similar calculation could be performed for density ramps of different shapes; while the precise relation between $S_{\text {eff }}$ and $L$ will be different, the core result that increasing $L$ reduces the apparent value of $S$ will still hold.

This result leads us to expect the properties of radiation emitted from a preplasma with given $S_{\text {eff }}$ to match those of radiation emitted from plasma with a sharp density profile, if the density in the latter case satisfies $S=S_{\text {eff }}$. We demonstrate 

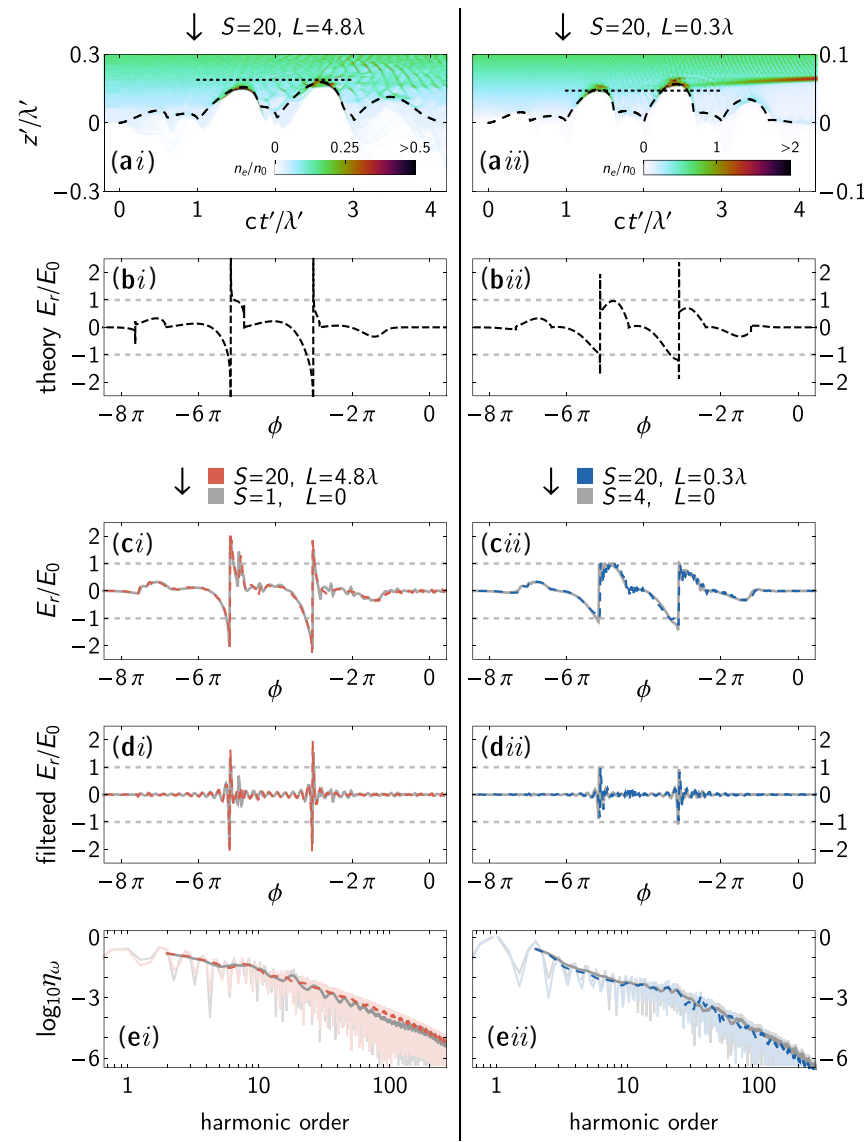

FIG. 4. XUV generation in laser illumination of plasma with a linear density ramp: (a) the electron density in the boosted frame (color scale), the maximum displacement predicted by Eq. (4) (dotted), and the RES model prediction of the electron displacement (dashed); (b) the RES model prediction for the laboratory-frame electric-field amplitude $E_{r}$ (relative to the peak initial amplitude $E_{0}$ ); (c) $E_{r} / E_{0}$ from simulation of a density ramp (colors), compared to the equivalent preplasma-free scenario (gray); (d) as in (b), but filtered to the XUV frequency range; (e) the spectral conversion efficiency: full (lighter colors) and smoothed over the width of a single harmonic (darker colors).

this for two exemplary cases, shown in Fig. 4. We consider plasma with bulk $S=20$ and preplasma scale lengths $L=4.8$ and $0.3 \mu \mathrm{m}$, corresponding to $S_{\text {eff }}=1$ and 4 , respectively. The laser and other simulation parameters are identical to those given in Sec. II.

First we verify that, in both cases, the maximum displacement of the electrons $z_{\max }^{\prime}$ is smaller than the preplasma scale length. This can be seen in Fig. 4(a), which shows the electron density in the boosted frame as a function of time $t^{\prime}$ and longitudinal displacement $z^{\prime}$. We also find that our simple prediction for $z_{\max }^{\prime}$ [Eq. (4)] agrees reasonably well with the simulation results; furthermore, the instantaneous displacement of the plasma-vacuum interface is modeled well by numerical solution of the RES equations [as given by Eq. (7) in [28]]. The fact that the laser pulse is reflected within the density ramp means that, as far as XUV generation is concerned, the interaction occurs in a plasma of much lower density than the bulk value of $S$ would imply.
The equivalence between the preplasma and preplasma-free cases suggested by Eq. (5) is evident when we compare the waveforms of the emitted radiation. Figure 4(c) shows excellent agreement between the electric-field amplitudes obtained from simulation of these two scenarios, provided that the density in the preplasma-free case is chosen according to Eq. (5). This is true even when the fields are filtered to the XUV frequency range, as shown in Fig. 4(d). (The results from the preplasma-free case have been translated in time to aid the eye.) The total $E_{r}$ predicted by solution of the RES equations for plasma with a linear density ramp is shown in Fig. 4(b). Aside from the singularities that also affected the comparison in Fig. 3(b), the simulation results are reproduced well.

Figure 4(e) shows that the spectral conversion efficiencies from simulation of preplasma and equivalent preplasma-free targets agree well across the XUV frequency range (harmonic orders between 10 and 100). As a result, the total XUV conversion efficiencies $\eta_{\mathrm{XUV}}$ are consistent between the preplasma and preplasma-free scenarios: for a plasma slab with bulk density $S=1(4), \eta_{\mathrm{XUV}}=19.2 \%(6.73 \%)$, whereas for a linear density ramp with $S_{\text {eff }}=1(4), \eta_{\text {XUV }}=19.9 \%$ (6.27\%). Note that XUV generation is more efficient for lower $S$, as we discussed in Sec. II, and for lower $S_{\text {eff }}$. Similarly, the peak electric-field amplitude of the reemitted radiation is larger for lower $S$ and for lower $S_{\text {eff }}$, leading us to expect an increased effective $a_{0}$. We will discuss the dependence of $\eta_{\mathrm{XUV}}$ and $a_{\mathrm{eff}}$ on the interaction parameters in the next section.

\section{OPTIMAL DENSITY, ANGLE OF INCIDENCE, AND PREPLASMA SCALE LENGTH}

We now discuss how laser-plasma interaction parameters may be chosen to maximize the increase in reflected electricfield amplitude and the yield of high harmonics. Previous work indicates that, for single-wavelength light and a perfectly sharp plasma-vacuum boundary, the optimal conditions are $S \simeq 1$ and $\theta \simeq 60^{\circ}$ [15]. Here we extend that analysis to the experimentally relevant scenario where there is a preplasma in front of the bulk, with parameter scans in laser intensity $I_{0}$, plasma density $n_{e}$, angle of incidence $\theta$, and preplasma scale length $L$ using $1 \mathrm{D}$ boosted-frame PIC simulations using the spectral code ELMIS [46]. The covered range is $10^{20} \mathrm{~W} \mathrm{~cm}^{-2} \leqslant$ $I_{0} \leqslant 10^{22} \mathrm{~W} \mathrm{~cm}^{-2}, 0.25 \leqslant S \leqslant 16$, and $0^{\circ}<\theta<80^{\circ}$. The laser duration is increased to $15 \mathrm{fs}$, i.e., its electric field as a function of phase is $\left(a_{0} m c \omega / e\right) \sin \phi \cos ^{2}(\phi / 24)$ for $|\phi|<$ $12 \pi$. Otherwise, the simulation parameters are as described in Sec. II.

Let us first consider the situation where $S$ is fixed to be 1 , the target is preplasma free, and the intensity and angle of incidence are varied. Here the electron density $n_{e}$ is implicitly increased with intensity as required by Eq. (1). Results are given in Figs. 5(a) and 5(b). We see that $a_{\text {eff }}>2$ for intensities $\gtrsim 5 \times 10^{20} \mathrm{~W} \mathrm{~cm}^{-2}$ provided that the angle of incidence $\theta \simeq$ $60^{\circ}$. For angles away from this optimum the electric-field amplitude increase associated with the RES mechanism is less efficient and $a_{\text {eff }}$ falls. This can be partially compensated by increasing the incident intensity; thus the acceptable range of incidence angles broadens with increasing intensity.

We may strip out the dependence on incident intensity by considering the conversion efficiency $\eta_{\mathrm{XUV}}$, as this is 

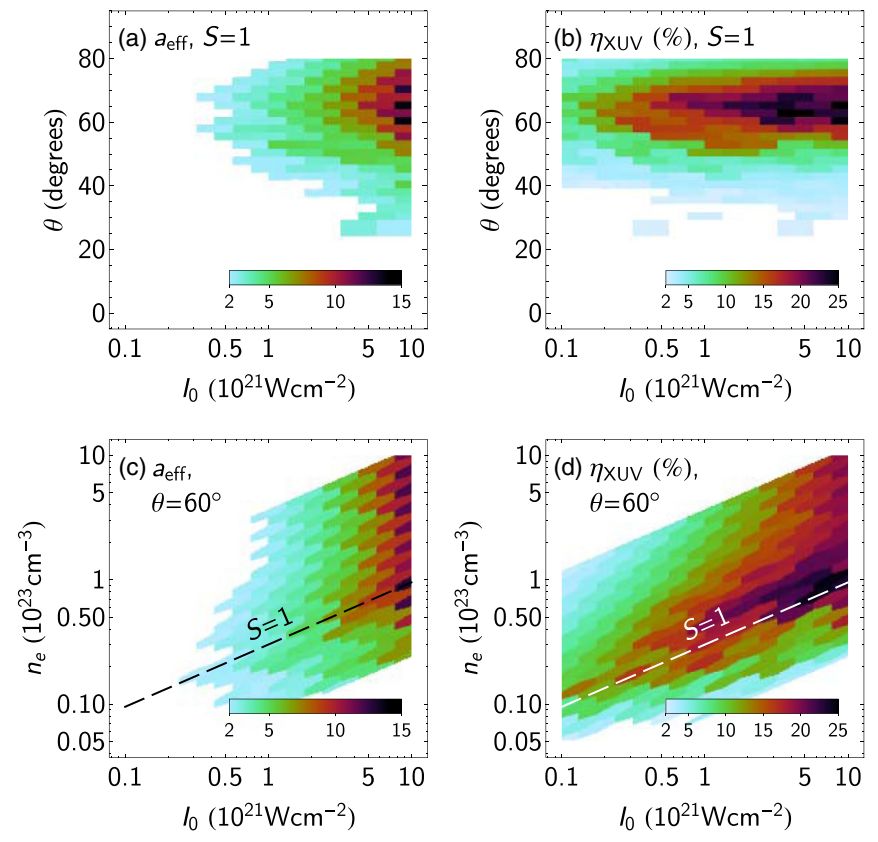

FIG. 5. (a), (c) Effective $a_{0}$ of, and (b), (d) the conversion efficiency to, XUV light when plasma with electron density $n_{e}=S a_{0} n_{\mathrm{cr}}$ is illuminated a laser pulse with peak intensity $I_{0}$, duration $15 \mathrm{fs}$, and wavelength $1 \mu \mathrm{m}$ at an angle $\theta$ to the target normal. For (a), (b) we fix $S=1$ and for (c), (d) $\theta=60^{\circ} . L=0$ for all.

normalized to the energy of the incident laser pulse. Figure 5(b) shows that $\eta_{\mathrm{XUV}}$ does not demonstrate a strong dependence on intensity for $I_{0}>5 \times 10^{20} \mathrm{~W} \mathrm{~cm}^{-2}$, which is consistent with the $S$ scaling [44]. There is little XUV emission for $\theta \lesssim 20^{\circ}$ but more than $15 \%$ of the pulse is converted for $55^{\circ} \gtrsim \theta \lesssim 75^{\circ}$. This maximum is consistent with that expected theoretically [15].

Fixing the angle of incidence at $60^{\circ}$, we explore intensitydensity space in Figs. 5(c) and 5(d). Increasing the plasma density at constant intensity, i.e., increasing $S$, decreases $a_{\text {eff }}$ as we expect given the results of Sec. II. As the incident intensity increases, so does $a_{\text {eff }}$ and the range of $S$ that ensures $a_{\text {eff }}>2$. The XUV conversion efficiency is largest for $S \gtrsim 0.5$ and decreases as $S$ increases. To clarify this point, we show the dependence of $a_{\text {eff }}$ and $\eta_{\mathrm{XUV}}$ on increasing $S$ for fixed laser intensities $(0.1,1,3,10) \times 10^{21} \mathrm{~W} \mathrm{~cm}^{-2}$ in Figs. 6(a) and 6(b), i.e., lineouts of Figs. 5(c) and 5(d) in the vertical direction. Both $a_{\text {eff }}$ and $\eta_{\mathrm{XUV}}$ have been scaled by the maximum value that is achieved at that particular intensity, namely $\max \left(a_{\mathrm{eff}}\right)=$ $(0.77,3.5,6.8,13)$ and $\max \left(\eta_{\mathrm{XUV}}\right)=(8.8,18,21,22) \%$. The curves are qualitatively similar in that $a_{\mathrm{eff}}$ and $\eta_{\mathrm{XUV}}$ increase as $S$ is increased up to $S \simeq 1$. Thereafter, they begin to decrease, with a gradient that becomes shallower at higher intensities. The range of optimal $S$ broadens to the extent that, at $10^{22} \mathrm{~W} \mathrm{~cm}^{-2}, a_{\text {eff }}$ is essentially constant for $S>2$. This violation of the $S$ similarity scaling is caused by ion motion, which has been studied in the context of increased broadening of high harmonics at normal incidence [50]. While such effects will be present here, as the ion longitudinal velocity and displacement are reduced at oblique incidence, the principal effect is that breakup of the plasma surface is enhanced, reducing the $S$ parameter accordingly.
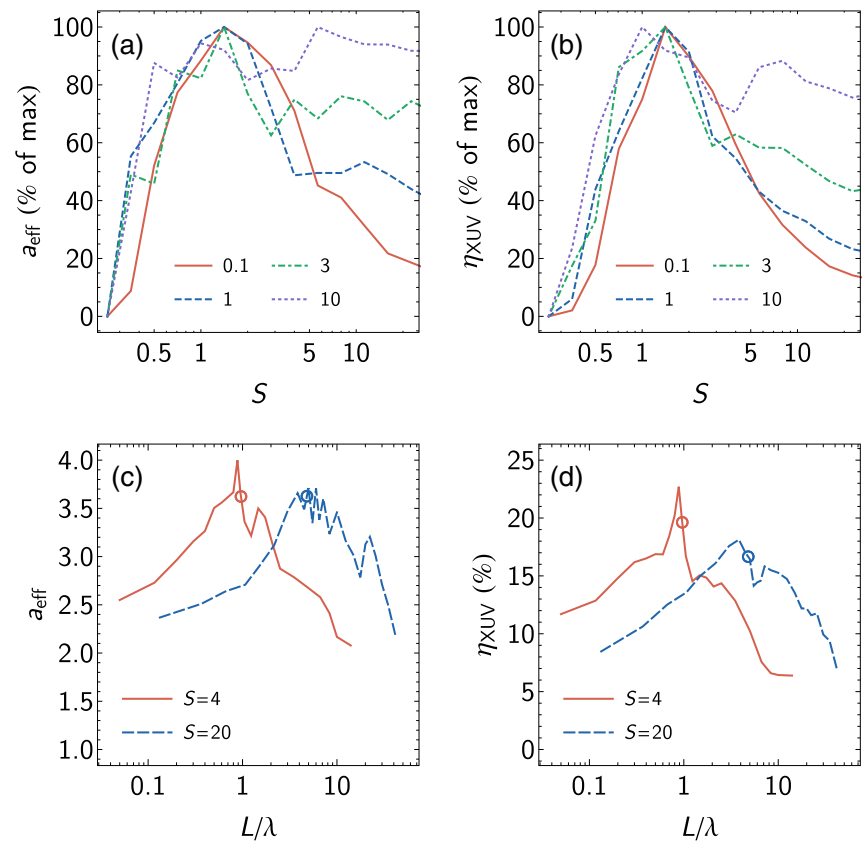

FIG. 6. (a) Effective $a_{0}$ and (b) conversion efficiency to XUV when plasma is illuminated at $60^{\circ}$ by a laser pulse with peak intensity $(0.1,1,3,10) \times 10^{21} \mathrm{~W} \mathrm{~cm}^{-2}$. Both $a_{\text {eff }}$ and $\eta_{\text {XUv }}$ have been scaled by the maximum value obtained at that intensity. The (c) effective $a_{0}$ and (d) conversion efficiency to XUV when plasma with preplasma scale length $L$ and bulk $S$ parameter is illuminated at $60^{\circ}$ by a laser pulse with peak intensity $10^{21} \mathrm{~W} \mathrm{~cm}^{-2}$. Circles indicate the $L$ for which $S_{\text {eff }}=1$ according to Eq. (5).

In Sec. II we saw that the presence of a density ramp in front of the target lowers the effective $S$ encountered by the laser pulse, permitting efficient XUV emission via the RES mechanism even for plasma with bulk $S \gg 1$. Figures 6(c) and 6(d) show that $a_{\text {eff }}$ and $\eta_{\mathrm{Xuv}}$ increase with increasing scale length $L$ up to a certain point and decrease thereafter. The open circles show that the locations of these maxima are consistent with the $L$ for which $S_{\text {eff }}=1$. Furthermore, the values of $\eta_{\mathrm{XUV}}$ for these $L$, approximately $17 \%$ for both $S=4$ and 20 , are consistent with that which would be obtained from an "ideal" target that has $S=1$ and is free of preplasma. Over the range of $L$ shown, the normalized amplitude and conversion efficiency are at least half this value. It is evident that the largest allowable $L$ grows with increasing $S$, as a longer density ramp is needed to reduce the effective $S$ parameter to the point that XUV generation is degraded. Taking the lower bound for $S_{\text {eff }}$ to be $\sim 0.25$, following the results shown in Fig. 5 , we can use Eq. (5) to estimate the largest allowable preplasma scale length $L_{\max } \simeq 3.8 S \lambda$ at $60^{\circ}$. This is consistent with the ranges shown in Figs. 6(c) and 6(d).

This result demonstrates the robustness of the RES mechanism and validates the use of an effective $S$ parameter to characterize the laser-plasma interaction. Expansion of the target due to prepulse heating, if well characterized and controlled, can be an aid rather than an obstacle to XUV generation by lowering the effective $S$ of the interaction. 


\section{DIVERGENCE, BRILLIANCE, AND INTENSITY}

To determine the angular divergence and brilliance of the XUV pulse so generated, we have carried out a parameter scan over $0.5 \leqslant S<15$ and $40^{\circ} \leqslant \theta \leqslant 75^{\circ}$ at fixed intensity $I_{0}=5 \times 10^{21} \mathrm{~W} \mathrm{~cm}^{-2}$ using the 2D3V PIC code EPOCH [51]. The laser pulse is plane polarized and focused to a spot size of $w_{0}=4 \mu \mathrm{m}$. Its temporal profile and wavelength are the same as in the 1D simulations: $\cos ^{2}$ with FWHM duration $15 \mathrm{fs}$ and $\lambda=1 \mu \mathrm{m}$, respectively. The simulation domain, $[0,16] \times$ $[-3,24] \lambda$ in the $x$ and $z$ directions, contains electron-ion plasma $(Z / A=0.5)$ in the region $z \leqslant 0$ with initial temperature $100 \mathrm{eV}$. The resolution varies between 200 and 500 cells per $\lambda$, and the number of particles per cell between 24 and 48 for each species. The laser is injected from the left boundary at an angle $\theta$ to the target normal; to reduce the length of plasma, and so the number of computational particles, required to model the interaction, the simulation domain moves along $x$ with velocity $c \sin \theta$. The electric-field amplitude and harmonic content of the reflected light is analyzed once the pulse has moved a perpendicular distance of $12 \mu \mathrm{m}$ from the plasma surface. Preplasma is added in the same way as for the 1D simulations, by including a linear density ramp of length $L$ in the $z$ direction in front of the bulk plasma. The position of the laser focus is $z=0$ : in the absence of preplasma, this corresponds to the plasma surface; in its presence, this is the depth at which the local density rises to $n_{e}(z=0)=a_{0} n_{\mathrm{cr}}$. The latter is motivated by our finding in Sec. III that the incident laser only penetrates the density ramp up to the point where the local density is approximately the relativistically critical density.

In moving from one to two spatial dimensions we encounter two new physical effects that alter the XUV generation process. The first is that the conservation of transverse kinetic momentum, which applies exactly in 1D, is lifted, leading to increased electron heating and reduction of the plasma reflectivity. Secondly, the variation in laser intensity across the plasma surface (or, equivalently, the variation in the effective $S$ ) leads to a spatially varying displacement of the electron-ion boundary and to wave-front curvature of the reflected light. Both of these effects are more pronounced for plasma of lower $S$ and are manifest, for example, in the increased ejection of electron bunches from the plasma surface. The peak density of the bunches is equivalent to at most $S \sim 0.5$ for the simulations presented here, slightly larger than the $S \sim 0.1$ for the $1 \mathrm{D}$ simulations in Sec. IV (ejection is negligible for the simulations in Secs. II and III). The amount of laser energy carried by these electrons is also larger in 2D than in $1 \mathrm{D}(\sim 2 \%$ and $\sim 1 \%$, respectively, regardless of preplasma), though a small fraction of the total.

In Fig. 7 we show the reemitted pulse electric-field amplitude from 2D simulations for various $S$ and $\theta$. For the lowest density, Fig. 7(a), the wave fronts of the leading edge are almost flat and they travel along the specular direction. Behind this, however, the wave fronts become strongly distorted as the pulse reflects from a curved plasma surface. The line out along the specular direction shows that the peak electric-field amplitude is approximately equal to that of the incident pulse, so the amplitude increase we expect from the RES mechanism is lost.

These effects are mitigated by moving to higher $S$. For $S=4$, Fig. 7(b) shows that the wave fronts are flat across
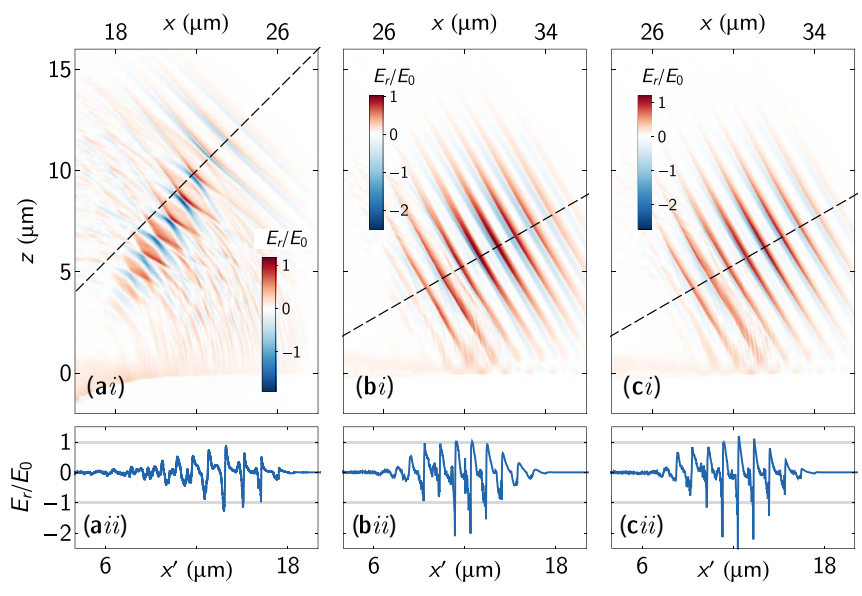

FIG. 7. (Upper) Color maps and (lower) line outs along the specular direction of the electric-field amplitude of the reemitted pulse when plasma with bulk $S$ and preplasma scale length $L$ is illuminated at angle $\theta$ by a laser pulse with peak intensity $5 \times 10^{21} \mathrm{~W} \mathrm{~cm}^{-2}$, where (a) $S=0.5, L=0, \theta=45^{\circ}$, (b) $S=4, L=0, \theta=60^{\circ}$, and (c) $S=4, L=0.3 \mu \mathrm{m}, \theta=60^{\circ}$.

the entire pulse and the peak electric-field amplitude is twice that of the incident pulse. If a linear density ramp with scale length $0.3 \mu \mathrm{m}$ is introduced in front of the bulk plasma, as shown in Fig. 7(c), we find that the peak electric-field amplitude is even larger, at $2.7 \times$ the initial value. This corresponds to achieving a peak intensity of $3.6 \times 10^{22} \mathrm{~W} \mathrm{~cm}^{-2}$, above the current record [47]. It should be noted that this increase arises in absence of any focusing, as the plasma surface has not been specially curved nor is there appreciable ponderomotive denting. It has been proposed that shaped plasma targets may be used to focus high harmonics to extreme intensity, exploiting the reduction in the diffraction limit $[52,53]$; such effects would stand in addition to the intensity increase arising from the RES mechanism that we show here.

The consequence of increased electron heating and nonuniformity of the plasma surface is to shift the optimum for XUV generation from $S=1$ to $S \simeq 4$, and reduce both $a_{\text {eff }}$ and the conversion efficiency $\eta_{\mathrm{XUV}}$. Nevertheless, the dependence of the former on $S$ and $\theta$ in the 2D case, shown in Fig. 8(a), demonstrates qualitative agreement with that found with $1 \mathrm{D}$ simulations. The reflected pulse is relativistically intense in the XUV frequency range for a broad range of angles around $60^{\circ}$ and $S \simeq 3$. Similarly, the XUV reflectivity shown in Fig. 8(b) still has the broad peak between $55^{\circ}$ and $75^{\circ}$ we expect based on 1D theory and simulation. The best value of $4 \%$ is approximately a factor of four smaller than that obtained in 1D. We attribute this to increased electron heating caused by wavelength-sized density perturbations, which are induced along the plasma surface by the oblique incidence of the laser pulse.

The final result of Sec. IV was that the effective $a_{0}$ and XUV conversion efficiency of plasmas with $S$ above the optimum could be improved by introducing a preplasma of sufficient length that the peak of the incoming pulse reflects off a surface with lower effective density. Figures 8(c) and 8(d) show that this principle still applies: the effective $a_{0}$ may be increased to $\sim 4$ and $\eta_{\mathrm{XUV}}$ to $5 \%$ for both $S=4$ and $S=20$. The preplasma 

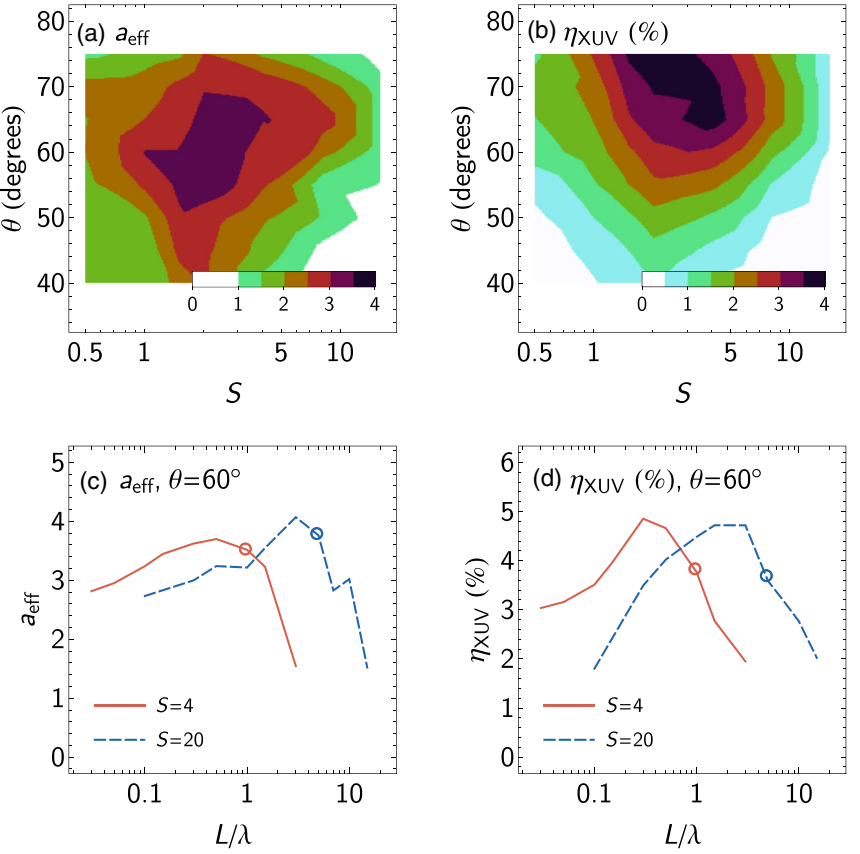

FIG. 8. (a) Effective $a_{0}$ and (b) the conversion efficiency in the XUV frequency range when plasma with electron density $n_{e}=S a_{0} n_{\mathrm{cr}}$ is illuminated by laser light with peak intensity $5 \times 10^{21} \mathrm{~W} \mathrm{~cm}^{-2}$ at angle $\theta$ to the target normal. (c) The effective $a_{0}$ and (d) XUV conversion efficiency when plasma with linear density ramp (scale length $L$ ) and bulk $S=4$ (blue, solid) and 20 (yellow, dashed) is illuminated at $60^{\circ}$ by a laser pulse with peak intensity $5 \times 10^{21} \mathrm{~W} \mathrm{~cm}^{-2}$. Circles indicate the $L$ for which $S_{\text {eff }}=1$, as predicted by Eq. (5).

scale length required is smaller than that which would make $S_{\text {eff }}=1$ according to Eq. (5); this is explained by the fact that, in $2 \mathrm{D}$, the optimal $S$ is larger and so the optimal $L$ must be reduced. Nevertheless, the qualitative behavior is as we find in $1 D$. We find that the generation of relativistically intense XUV light with good conversion efficiency is robust against the presence of preplasma over a wide range of scale lengths.

The flat wave fronts shown in Figs. 7(b) and 7(c) suggest that the emission of high harmonics is closely collimated along the specular direction, with angular divergence comparable to that of the incoming laser pulse. We show in Figs. 9(a), 9(b), and 9 (c) color maps of $\eta_{\omega, \Omega}$, the conversion efficiency per unit frequency $\omega$, and solid angle $\cos \theta$. The harmonics become more distinct, with smaller divergence, as $S$ increases. Slices at the 10th and 20th harmonics show that for $S=4$ the reflected energy is concentrated within a window $\Delta(\cos \theta) \simeq 0.05$, corresponding to a half-angle of $3^{\circ}$ at $60^{\circ}$. For comparison, the incident laser pulse with waist $w_{0}=4 \mu \mathrm{m}$ and wavelength $1 \mu \mathrm{m}$ has half-angle divergence $\theta_{\text {laser }}=\lambda /\left(\pi w_{0}\right) \simeq 5^{\circ}$. One might expect that the wavelength dependence in this relation leads to decreasing divergence with increasing harmonic order. However, Fig. 9 shows that the divergence of the 20th harmonic is near, not half, that of the 10th, and therefore the dominant effect is the overall divergence of the laser pulse. The distribution of energy in $\omega$ and $\cos \theta$ is broader and noisier for $S=0.5$ than it is for $S=4$. The broadening is caused by ponderomotive denting of the plasma surface, which increases the curvature of the reflected wave fronts, i.e., emission of high-

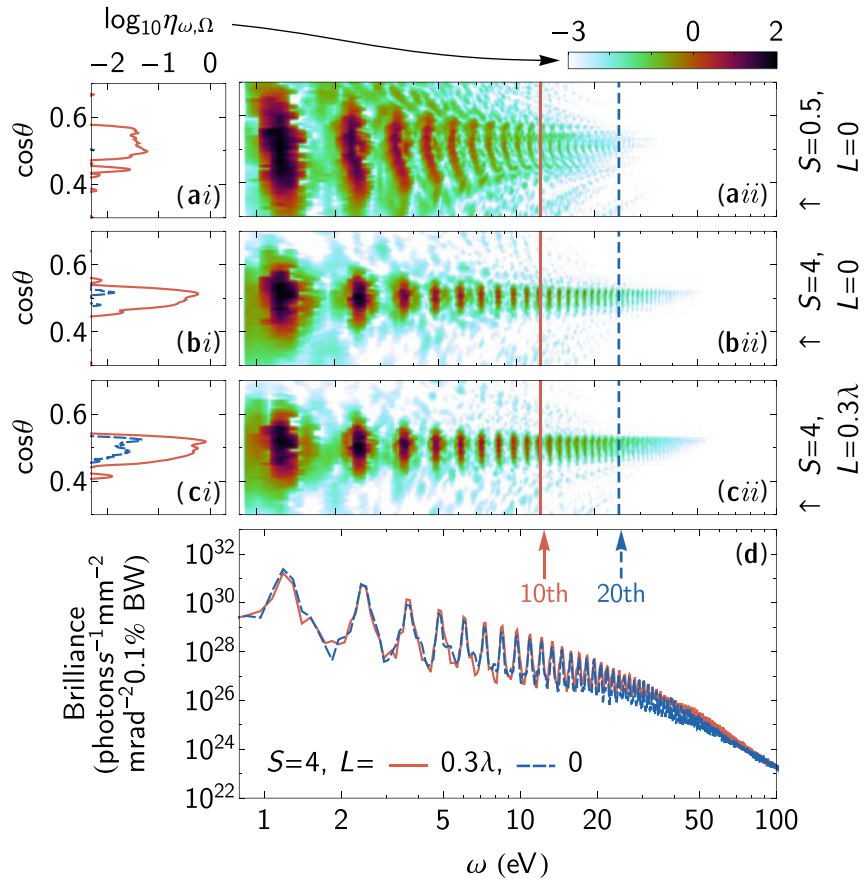

FIG. 9. (a)-(c) $\eta_{\omega, \Omega}$, the conversion efficiency per unit photon energy $\omega$ per unit solid angle $\cos \theta$, for plasma with given $S$ and preplasma scale length $L$ irradiated by a laser pulse with peak intensity $5 \times 10^{21} \mathrm{~W} \mathrm{~cm}^{-2}$ at incidence angle $60^{\circ}$, showing slices through color maps (ii) at the (blue, solid) 10th and (yellow, dashed) 20th harmonics. (d) The brilliance for $S=4$ and given $L$ at $60^{\circ}$.

frequency radiation away from the specular axis. Figures 9(b) and 9(c) differ only by the inclusion of preplasma, which does not affect the angular properties of the radiation aside from an overall increase in the total conversion efficiency.

Finally, we use the spectral conversion efficiency from simulation to estimate the brilliance, which is a measure of photon phase-space density. Assuming a fiducial distance in the $y$ direction (perpendicular to the simulation plane) of $4 \mu \mathrm{m}$ to determine the total energy of the pulse, a spot size of $\pi w_{0}^{2}$, a duration equal to the laser pulse of $15 \mathrm{fs}$, and a half-angle divergence of $3^{\circ}$, we show in Fig. 9 that the brilliance is of order $10^{23}$ photons $/ \mathrm{s} / \mathrm{mm}^{2} / \mathrm{mrad}^{2}(0.1 \%$ bandwidth) at $\omega=100 \mathrm{eV}$, for the optimal parameters of $S=4, \theta=60^{\circ}$ and a linear density ramp of preplasma with length $L=0.3 \mu \mathrm{m}$. While this is comparable to the brilliance achieved in third-generation synchrotron light sources [54], it is lower than that achieved by advanced gas harmonic sources [55], and six to nine orders of magnitude smaller than that reached by x-ray free-electron lasers [56]. The benefit of employing an ultraintense laser-plasma interaction to generate XUV radiation is that the larger pulse energy and femtosecond duration make it possible for the high harmonics to be both bright and relativistically intense, a unique capability of the near-critical interaction scenario presented here.

\section{CONCLUSIONS}

We have explored how the relativistic electron spring mechanism leads to bright, intense bursts of XUV radiation when plasma with electron density $n_{e}$ satisfying $1<S=n_{e} /$ 
$\left(a_{0} n_{\mathrm{cr}}\right)<10$ is illuminated by intense laser light $\left(a_{0} \gg 1\right)$. The physical origin of this enhanced emission is the storage of energy in plasma electromagnetic fields when the electron-ion boundary is displaced by the oscillating radiation pressure of the laser. We have justified the theoretical prediction of the optimal parameters $S=1, \theta=60^{\circ}$ with high-resolution 1D PIC simulation and shown further that the presence of preplasma can be beneficial by lowering the effective $S$ of a plasma with bulk density higher than would be optimal.

Parametric analysis with 2D simulations indicates that electron heating and ponderomotive denting of the plasma surface shift the optimal $S$ upwards to $S \simeq 4$. However, the consequent reduction in conversion efficiency can be partially offset by preplasma, permitting conversion efficiencies of $\sim 5 \%$ to XUV light collimated at the degree level even for $S=20$. The reemitted pulse can reach a peak intensity five times greater than the incident pulse, sufficient to be relativistically intense when filtered to the XUV frequency range. This capability provides opportunities for XUV-pump-XUV-probe experiments, exploiting the ultrashort duration of the pulse and the comparatively compact size of a high-intensity laser facility.

\section{ACKNOWLEDGMENTS}

The authors acknowledge support from the Knut and Alice Wallenberg Foundation (T.G.B., A.G., M.M.), the Swedish Research Council [Grants No. 2013-4248 and No. 201603329 (M.M.) and Grant No. 2017-05148 (A.G.)], the Russian Science Foundation [Project No. 16-12-10486 (A.G.), RES calculations], and the Russian Foundation for Basic Research [Project No. 15-37-21015 (A.G.)]. Simulations were performed on resources provided by the Swedish National Infrastructure for Computing (SNIC) at the High Performance Computing Centre North (HPC2N).
[1] A. Rousse, P. Audebert, J. P. Geindre, F. Falliès, J. C. Gauthier, A. Mysyrowicz, G. Grillon, and A. Antonetti, Phys. Rev. E 50, 2200 (1994).

[2] B. Dromey, M. Zepf, A. Gopal, K. Lancaster, M. S. Wei, K. Krushelnick, M. Tatarakis, N. Vakakis, S. Moustaizis, R. Kodama, M. Tampo, C. Stoeckl, R. Clarke, H. Habara, D. Neely, S. Karsch, and P. Norreys, Nat. Phys. 2, 456 (2006).

[3] F. Quéré, C. Thaury, P. Monot, S. Dobosz, P. Martin, J.-P. Geindre, and P. Audebert, Phys. Rev. Lett. 96, 125004 (2006).

[4] B. Dromey, D. Adams, R. Hörlein, Y. Nomura, S. G. Rykovanov, D. C. Carroll, P. S. Foster, S. Kar, K. Markey, P. McKenna, D. Neely, M. Geissler, G. D. Tsakiris, and M. Zepf, Nat. Phys. 5, 146 (2009).

[5] A. Rousse, K. T. Phuoc, R. Shah, A. Pukhov, E. Lefebvre, V. Malka, S. Kiselev, F. Burgy, J.-P. Rousseau, D. Umstadter, and D. Hulin, Phys. Rev. Lett. 93, 135005 (2004).

[6] S. Kneip, C. McGuffey, J. L. Martins, S. F. Martins, C. Bellei, V. Chvykov, F. Dollar, R. Fonseca, C. Huntington, G. Kalintchenko, A. Maksimchuk, S. P. D. Mangles, T. Matsuoka, S. R. Nagel, C. A. J. Palmer, J. Schreiber, K. Ta Phuoc, A. G. R. Thomas, V. Yanovsky, L. O. Silva, K. Krushelnick, and Z. Najmudin, Nat. Phys. 6, 980 (2010).

[7] S. Corde, K. Ta Phuoc, G. Lambert, R. Fitour, V. Malka, A. Rousse, A. Beck, and E. Lefebvre, Rev. Mod. Phys. 85, 1 (2013).

[8] S. Dobosz, G. Doumy, H. Stabile, P. D’Oliveira, P. Monot, F. Réau, S. Hüller, and P. Martin, Phys. Rev. Lett. 95, 025001 (2005).

[9] C. M. Brenner, S. R. Mirfayzi, D. R. Rusby, C. Armstrong, A. Alejo, L. A. Wilson, R. Clarke, H. Ahmed, N. M. H. Butler, D. Haddock, A. Higginson, A. McClymont, C. Murphy, M. Notley, P. Oliver, R. Allott, C. Hernandez-Gomez, S. Kar, P. McKenna, and D. Neely, Plasma Phys. Control. Fusion 58, 014039 (2016).

[10] B. Nagler et al., Nat. Phys. 5, 693 (2009).

[11] E. Galtier, F. B. Rosmej, T. Dzelzainis, D. Riley, F. Y. Khattak, P. Heimann, R. W. Lee, A. J. Nelson, S. M. Vinko, T. Whitcher, J. S. Wark, T. Tschentscher, S. Toleikis, R. R. Fäustlin, R. Sobierajski, M. Jurek, L. Juha, J. Chalupsky, V. Hajkova, M. Kozlova, J. Krzywinski, and B. Nagler, Phys. Rev. Lett. 106, 164801 (2011).
[12] F. Krausz and M. Ivanov, Rev. Mod. Phys. 81, 163 (2009).

[13] P. Tzallas, E. Skantzakis, L. A. A. Nikolopoulos, G. D. Tsakiris, and D. Charalambidis, Nat. Phys. 7, 781 (2011).

[14] D. an der Brügge and A. Pukhov, Phys. Plasmas 17, 033110 (2010).

[15] A. A. Gonoskov, A. V. Korzhimanov, A. V. Kim, M. Marklund, and A. M. Sergeev, Phys. Rev. E 84, 046403 (2011).

[16] U. Teubner and P. Gibbon, Rev. Mod. Phys. 81, 445 (2009).

[17] B. Dromey, S. Kar, C. Bellei, D. C. Carroll, R. J. Clarke, J. S. Green, S. Kneip, K. Markey, S. R. Nagel, P. T. Simpson, L. Willingale, P. McKenna, D. Neely, Z. Najmudin, K. Krushelnick, P. A. Norreys, and M. Zepf, Phys. Rev. Lett. 99, 085001 (2007).

[18] C. Rödel, D. an der Brügge, J. Bierbach, M. Yeung, T. Hahn, B. Dromey, S. Herzer, S. Fuchs, A. G. Pour, E. Eckner, M. Behmke, M. Cerchez, O. Jäckel, D. Hemmers, T. Toncian, M. C. Kaluza, A. Belyanin, G. Pretzler, O. Willi, A. Pukhov, M. Zepf, and G. G. Paulus, Phys. Rev. Lett. 109, 125002 (2012).

[19] S. V. Bulanov, N. M. Naumova, and F. Pegoraro, Phys. Plasmas 1, 745 (1994).

[20] R. Lichters, J. Meyer-ter-Vehn, and A. Pukhov, Phys. Plasmas 3, 3425 (1996).

[21] D. von der Linde and K. Rzàzewski, Appl. Phys. B 63, 499 (1996).

[22] A. Debayle, J. Sanz, and L. Gremillet, Phys. Rev. E 92, 053108 (2015).

[23] T. Baeva, S. Gordienko, and A. Pukhov, Phys. Rev. E 74, 046404 (2006).

[24] A. S. Pirozhkov, S. V. Bulanov, T. Z. Esirkepov, M. Mori, A. Sagisaka, and H. Daido, Phys. Plasmas 13, 013107 (2006).

[25] A. Debayle, J. Sanz, L. Gremillet, and K. Mima, Phys. Plasmas 20, 053107 (2013).

[26] T. J. M. Boyd and R. Ondarza-Rovira, Phys. Rev. Lett. 101, 125004 (2008).

[27] T. J. M. Boyd and R. Ondarza-Rovira, Phys. Lett. A 380, 1368 (2016).

[28] A. Gonoskov, Phys. Plasmas 25, 013108 (2018).

[29] B. Dromey, S. Cousens, S. Rykovanov, M. Yeung, D. Jung, D. C. Gautier, T. Dzelzainis, D. Kiefer, S. Palaniyppan, R. Shah, 
J. Schreiber, J. C. Fernandez, C. L. S. Lewis, M. Zepf, and B. M. Hegelich, New J. Phys. 15, 015025 (2013).

[30] G. Ma, W. Dallari, A. Borot, F. Krausz, W. Yu, G. D. Tsakiris, and L. Veisz, Phys. Plasmas 22, 033105 (2015).

[31] S. Cousens, B. Reville, B. Dromey, and M. Zepf, Phys. Rev. Lett. 116, 083901 (2016).

[32] Y. X. Zhang, B. Qiao, X. R. Xu, H. X. Chang, H. Zhang, H. Y. Lu, M. Y. Yu, C. T. Zhou, S. P. Zhu, and X. T. He, Phys. Plasmas 24, 123119 (2017).

[33] J. M. Mikhailova, M. V. Fedorov, N. Karpowicz, P. Gibbon, V. T. Platonenko, A. M. Zheltikov, and F. Krausz, Phys. Rev. Lett. 109, 245005 (2012).

[34] S. V. Bulanov, T. Z. Esirkepov, M. Kando, S. S. Bulanov, S. G. Rykovanov, and F. Pegoraro, Phys. Plasmas 20, 123114 (2013).

[35] A. V. Bashinov, A. A. Gonoskov, A. V. Kim, G. Mourou, and A. M. Sergeev, Eur. Phys. J. Spec. Top. 223, 1105 (2014).

[36] J. Fuchs, A. A. Gonoskov, M. Nakatsutsumi, W. Nazarov, F. Quéré, A. M. Sergeev, and X. Q. Yan, Eur. Phys. J. Spec. Top. 223, 1169 (2014).

[37] M. Blanco, M. T. Flores-Arias, and A. Gonoskov, arXiv:1706.04785 [physics.plasm-ph].

[38] D. A. Serebryakov, E. N. Nerush, and I. Yu. Kostyukov, Phys. Plasmas 22, 123119 (2015).

[39] G. Sansone, L. Poletto, and N. Mauro, Nat. Photon. 5, 655 (2011).

[40] G. D. Tsakiris, K. Eidmann, J. Meyer-ter-Vehn, and F. Krausz, New J. Phys. 8, 19 (2006).

[41] H. Vincenti, S. Monchocé, S. Kahaly, G. Bonnaud, Ph. Martin, and F. Quéré, Nat. Commun. 5, 3403 (2014).

[42] M. R. Edwards and J. M. Mikhailova, Phys. Rev. A 93, 023836 (2016).
[43] M. R. Edwards and J. M. Mikhailova, Phys. Rev. Lett. 117, 125001 (2016).

[44] S. Gordienko and A. Pukhov, Phys. Plasmas 12, 043109 (2005).

[45] A. Bourdier, Phys. Fluids 26, 1804 (1983).

[46] A. Gonoskov, S. Bastrakov, E. Efimenko, A. Ilderton, M. Marklund, I. Meyerov, A. Muraviev, A. Sergeev, I. Surmin, and E. Wallin, Phys. Rev. E 92, 023305 (2015).

[47] S.-W. Bahk, P. Rousseau, T. A. Planchon, V. Chvykov, G. Kalintchenko, A. Maksimchuk, G. A. Mourou, and V. Yanovsky, Opt. Lett. 29, 2837 (2004).

[48] B. S. Wettervik, A. Gonoskov, and M. Marklund, Phys. Plasmas 25, 013107 (2018).

[49] L. Fedeli, A. Formenti, C. E. Bottani, and M. Passoni, Eur. Phys. J. D 71, 202 (2017).

[50] S. Tang, N. Kumar, and C. H. Keitel, Phys. Rev. E 95, 051201 (2017).

[51] T. D. Arber, K. Bennett, C. S. Brady, A. Lawrence-Douglas, M. G. Ramsay, N. J. Sircombe, P. Gillies, R. G. Evans, H. Schmitz, A. R. Bell, and C. P. Ridgers, Plasma Phys. Control. Fusion 57, 1 (2015).

[52] N. M. Naumova, J. A. Nees, I. V. Sokolov, B. Hou, and G. A. Mourou, Phys. Rev. Lett. 92, 063902 (2004).

[53] S. Gordienko, A. Pukhov, O. Shorokhov, and T. Baeva, Phys. Rev. Lett. 94, 103903 (2005).

[54] The Advanced Light Source, Machine Information (Lawrence Berkeley National Laboratory, Berkeley, CA, 2016).

[55] D. Popmintchev, B. R. Galloway, M.-C. Chen, F. Dollar, C. A. Mancuso, A. Hankla, L. Miaja-Avila, G. O’Neil, J. M. Shaw, G. Fan, S. Ališauskas, G. Andriukaitis, T. Balčiunas, O. D. Mücke, A. Pugzlys, A. Baltuška, H. C. Kapteyn, T. Popmintchev, and M. M. Murnane, Phys. Rev. Lett. 120, 093002 (2018).

[56] W. Ackermann et al., Nat. Photon. 1, 336 (2007). 